\title{
A Wideband Metal-Only Transmitarray With Two-layer Configuration
}

\author{
Wei Hu, Jianshen Dong, Qi Luo, Senior Member, IEEE, Yuanming Cai, Xuekang Liu, Lehu Wen, \\ Wen Jiang, and Steven Gao, Fellow, IEEE
}

\begin{abstract}
This letter presents a novel wideband metal-only transmitarray (TA). Up to date, existing works on metal-only TAs use at least three layers spaced by air gaps. Compared to these designs, the presented TA has only two layers and can realize a full $360^{\circ}$ phase shift with low insertion loss and wide bandwidth. The TA unit cell consists of two metal layers connected by a metal post and converts the linearly polarized incident wave to the orthogonal direction. This type of configuration increases the mechanical robustness of the TA surface and improves the alignment accuracy of the layers. Through mirroring the slots on the bottom layer, the electrical field of the transmitted wave is rotated by $180^{\circ}$ which is equivalent to a phase shift of $180^{\circ}$. Using this characteristic and simultaneously adjusting the lengths of the slots, a continuously $360^{\circ}$ phase shift range is obtained. To verify this design concept, a prototype consists of 256-unit cells was designed, fabricated, and measured. The experimental results show that the TA has $14.6 \%$ 1-dB gain bandwidth and $47.6 \%$ aperture efficiency at $8.5 \mathrm{GHz}$. The proposed TA has the advantages of wideband, no dielectric loss, and robust performance.
\end{abstract}

Index Terms-Metal-only, transmitarray (TA), wideband.

\section{INTRODUCTION}

$\mathrm{T}$ RANSMITARRAY (TA) combines the features of lens and phased array antenna [1]. Because of its advantages such as high gain, planar structure, and free from feed blockage, TA is widely used in satellite communication systems and radar systems [2], [3]. Generally speaking, there are two traditional methods for TA design. The first method is to employ the "receive-transmit" antenna configuration. In this way, the phase shift can be achieved by changing the relative position of the transmitting antenna of the unit cells [4]-[8] or changing the length of the phase delay lines [9], [10]. Another method for designing TAs is based on the frequency selective surfaces or metasurfaces [11]-[17].

Recently, the metal-only TA has attracted many research interests. The metal-only structure means that there would be

This work was supported in part by the 111 Project of China, in part by EPSRC under Grants EP/N032497/1 and EP/S005625/1, and in part by the National Natural Science Foundation of China under Grant No. 62001348.

W. Hu, J. Dong, Y. Cai and W. Jiang are with the National Key Laboratory of Antennas and Microwave Technology, Xidian University, Xi'an, Shaanxi 710071, China. (e-mail: weihu.xidian@ieee.org).

Q. Luo is with the Department of Engineering and Technology, University of Hertfordshire, AL10 9AB, U.K. (e-mail: qiluo@ieee.org).

X. Liu, L. Wen and S. Gao are with the School of Engineering and Digital Arts, University of Kent, Canterbury CT2 7NT, U.K. (e-mail: s.gao@kent.ac.uk ). no dielectric loss and the antenna would show stable performance in more stringent environments [12]-[17]. For the best knowledge of the authors, up to date, existing works on metal-only TAs use at least three layers. In [12], a four-layer metal-only TA using slot-type unit cell obtains a 1-dB gain bandwidth of $4.2 \%$. A metal-only TA panel with a four-layer configuration is developed in [15] for mode transducing, and a 9.7\% 1-dB gain bandwidth is achieved. In [16], TA with three layers achieved wide 1-dB gain bandwidth of $15.5 \%$. In [17], polarization manipulation is used in a $\mathrm{C}$-shaped unit cell. The achieved $3-\mathrm{dB}$ gain bandwidth of the TA is $11 \%$. These reported metal-only designs use air gaps to separate each layer. When the size of the aperture is large, the mechanical robustness of the TA surface becomes a challenging issue. Although some two layers TA are designed using PCB [18], [19], they are not suitable for metal-only TA because each TA element needs to be fabricated individually, which leads to high fabrication and assembling complexity.

This work aims to fill this gap by investigating the design of a metal-only TA using only two layers while achieving wide operation bandwidth, low insertion loss, good mechanical robustness, and low fabrication complexity. To obtain a wider passband, each layer of unit cell uses two slot resonators of different lengths, corresponding to different resonant frequencies. The two metal layers of the unit cell are etched with identical slots, but the slots on different layers are placed orthogonally. A metal post is used to connect these two metal layers. Besides serving as the interconnection, the metal post also works as a supporting spacer, which makes the developed TA easy to assemble with high alignment accuracy and good mechanical strength. The proposed unit cell twists the transmitted wave by $-90^{\circ}$ or $90^{\circ}$ compared with the linearly polarized incident wave. Based on this characteristic, the transmitted wave provides a $0^{\circ} / 180^{\circ}$ phase shift. By varying the length of the slots, another continuous $180^{\circ}$ phase shift is obtained. Thus, a continuous $360^{\circ}$ phase shift is realized.

\section{UNIT CELl DESIGN}

The configuration of the proposed TA unit cell is shown in Fig. 1(a). The metal-only unit cell consists of two layers and a metal post connecting the center of the two layers. There are two U-shaped slots of different lengths etched in each metal layer, located symmetrically with respect to the center of the surface. The layouts of the slots in the top layer and bottom layer are given in Fig.1(b) and Fig.1(c). The slots on the top and 


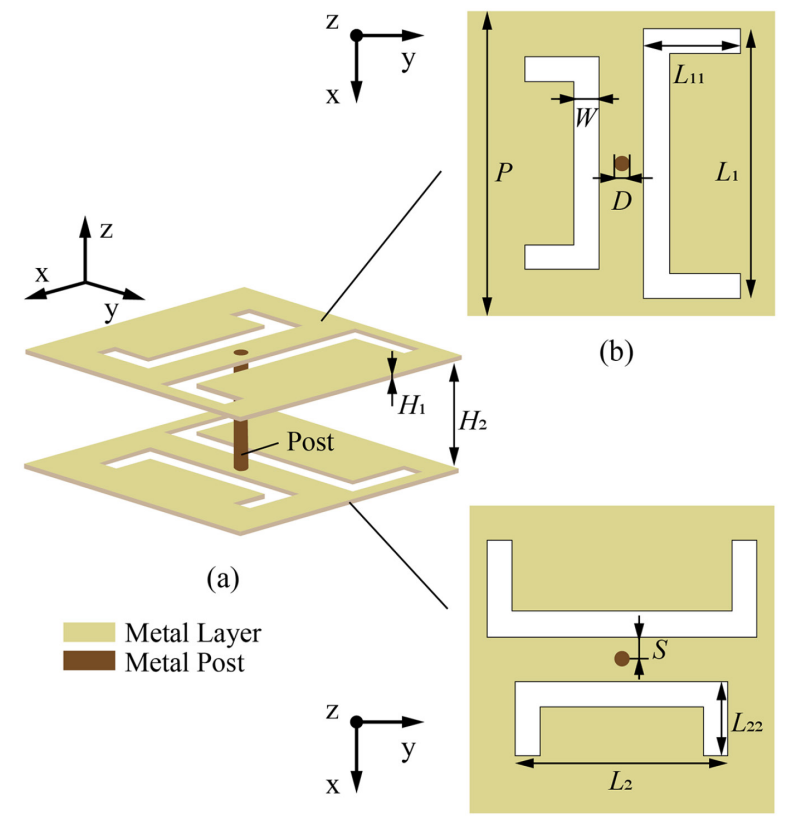

(c)

Fig. 1. Geometry of the unit cell. (a) 3D structure. (b) Top view. (c) Bottom view. Dimensions are $P=18, H_{1}=1, H_{2}=6, W=1.5, D=0.8, S=1.3, L_{1}=$ 13.4-16.2, $L_{11}=0.35 L_{1}, L_{2}=0.79 L_{1}, L_{22}=0.35 L_{2}$ (unit: mm).

bottom layers are the same and the only difference is that the slots in the two layers are placed orthogonally. The long side of the slots in the upper metal layer is parallel to the $x$-axis, and the long side of the slots in the bottom metal layer is parallel to the $y$-axis. To reduce the size of the unit cell, the slots are bent into U-shapes. To enhance the coupling between the slots in the top and bottom layers, a metal post is introduced. As a result of using two different U-shaped slots, the unit cell has a wide passband, which is critical to realize a wideband TA design. The spacing between the two layers was initially chosen as quarter wavelength and then optimized to $6 \mathrm{~mm}$. The electromagnetic wave is received by one layer and then transmitted to the other layer. Due to its orthogonal structure, the unit cell twists the polarization of the transmitted wave by $\pm 90^{\circ}$ compared to the linearly polarized incident wave.

The material of the metal layers and metal post is brass with a conductivity of $1.5 \times 10^{7} \mathrm{~S} / \mathrm{m}$. The operating frequency of the unit cell is $8.5 \mathrm{GHz}$, and the spacing of the unit cell is $18 \mathrm{~mm}$ $\left(0.52 \lambda_{0}\right.$, where $\lambda_{0}$ is the free space wavelength at $\left.8.5 \mathrm{GHz}\right)$. The transmission responses of the unit cell are simulated using Floquet method analysis in ANSYS HFSS. The TA unit cell shown in Fig. 1 has good polarization conversion performance. When the electromagnetic wave transmits in the -z-direction, the polarization conversion is shown in Fig. 2. The unit cell can efficiently convert $y$-polarized wave into $x$-polarized wave in the frequency band of interest. In this design, to achieve a continuous $360^{\circ}$ phase shift while maintaining a wide passband range of the unit cell, two methods are used to shift the phase of the TA unit cell.

1) mirror the position of the slots: by rotating the slots in the bottom layer by $180^{\circ}$, the phase of the transmitted signal can be changed by $180^{\circ}$. To better understand the phase difference

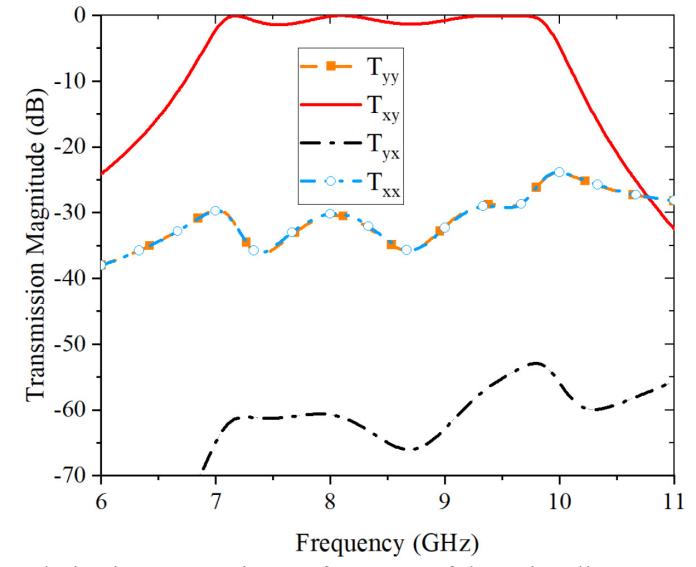

Fig. 2. Polarization conversion performance of the unit cell.

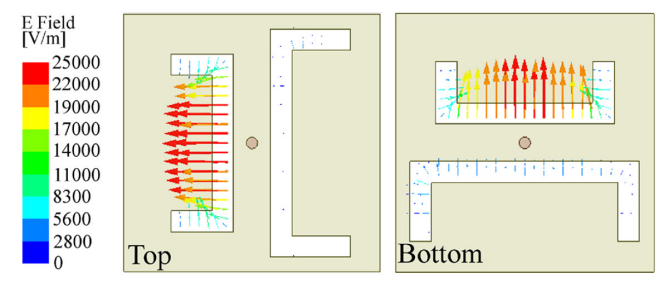

(a)

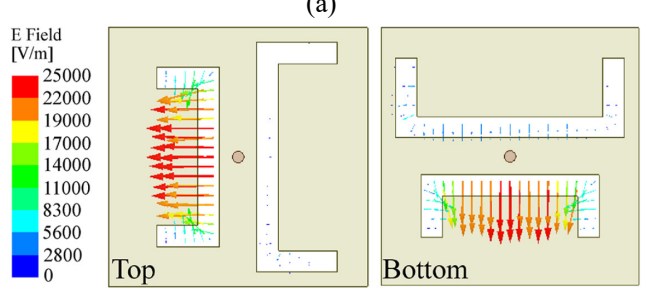

(b)

Fig. 3. Distribution of the electric field in the top and bottom slots at $8.5 \mathrm{GHz}$. (a)State A, (b)State B.

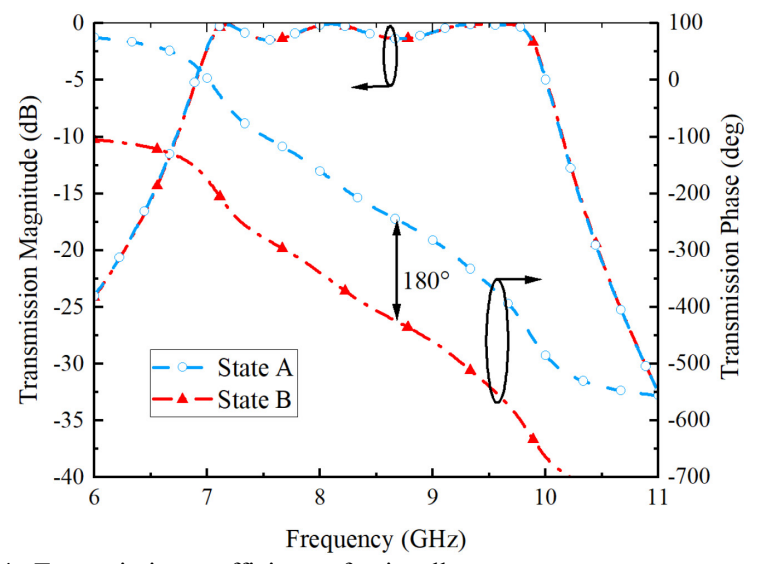

Fig. 4. Transmission coefficients of unit cell states.

between state $\mathrm{A}$ and state $\mathrm{B}$ of the TA unit cell, the electric field in the slots of these two states are shown in Fig. 3. Because of the reversal electric field (the slots in the bottom layer are mirrored), the two states of the unit cell have a $180^{\circ}$ phase difference. Fig. 4 shows the transmission magnitude and phase response of these two states. The two states have the same magnitude of transmission, but with $180^{\circ}$ phase differences.

2) change the length of the slots: Since the resonant frequency of the unit cell is determined by the lengths of the slots, changing the lengths of the slots adjust the phase shift of 


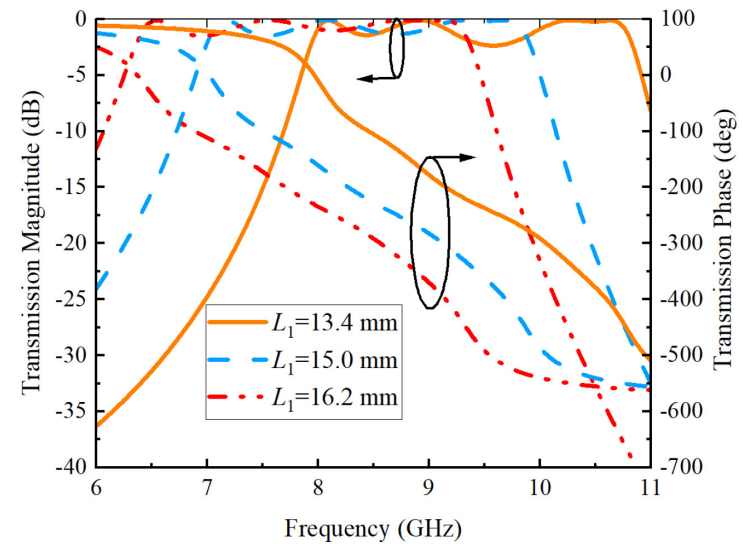

Fig. 5. Magnitude and phase responses of the transmission coefficients with varied values of $L_{1}$ at state $\mathrm{A}$.

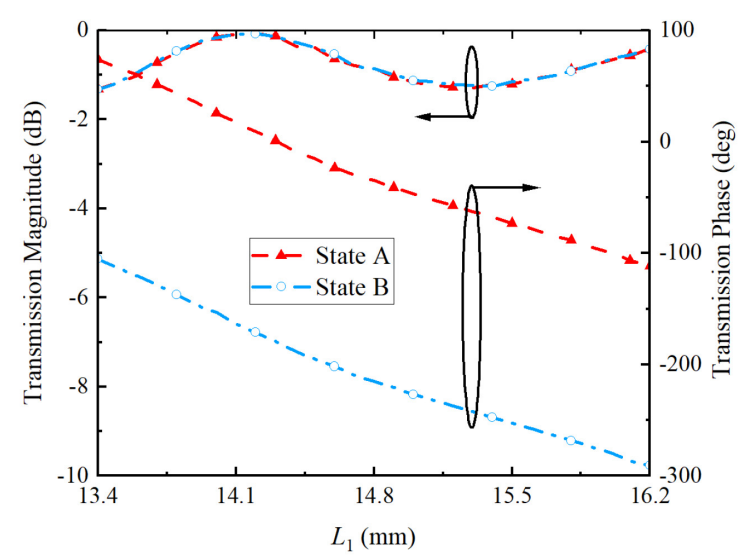

Fig. 6. Variation of the transmission coefficients of the unit cell as a function of the slot length $L_{1}$ at $8.5 \mathrm{GHz}$.

the TA unit cell. In this design, all slots of unit cell are varied with $L_{1}$ proportionally. Fig. 5 shows the simulated magnitude and phase responses of the transmission coefficients when changing the value of $L_{1}$. As shown, when varying $L_{1}$ from 13.4 to $16.2 \mathrm{~mm}$, the unit cell provides $180^{\circ}$ continuous phase shift, while the passband consistently maintains less than $2 \mathrm{~dB}$ transmission loss from 7.95 to $9.35 \mathrm{GHz}$. Fig. 6 shows the $360^{\circ}$ phase shift is obtained by combining these two methods when the transmission coefficients of the unit cell as a function of $L_{1}$, and the insertion loss is less than $1.4 \mathrm{~dB}$ at $8.5 \mathrm{GHz}$.

\section{TRANSMITARRAY DESIGN AND RESUlTS}

Using the developed unit cell, a TA with a square aperture and 256-unit cells is designed. A linearly polarized horn was used as the feed and is placed $289 \mathrm{~mm}$ below the center of the array. The $F / D$ ratio is 1 where $F$ is the distance from the feed's phase center to the center of the TA panel and $D$ is the width of the panel. The waveguide of the horn is WR112. The horn has a gain of $16.3 \mathrm{dBi}$ at $8.5 \mathrm{GHz}$ and its aperture is $60 \mathrm{~mm} \times 85 \mathrm{~mm}$.

As discussed in the previous section, two methods are simultaneously used to adjust the phase shift of each unit cell. After the relative phase distribution of the TA aperture is calculated, the unit cells are divided into two groups. One group has phase shifts in the range of $\left[0-180^{\circ}\right]$, and the other group has phase shifts in the range of $\left[180^{\circ}-360^{\circ}\right]$. Then, the value of

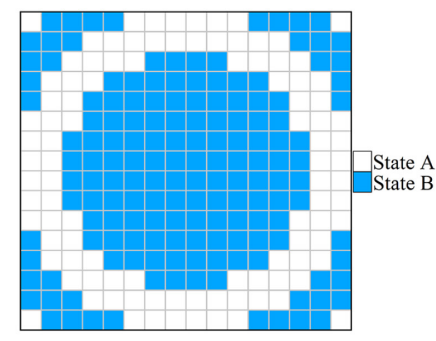

(a)

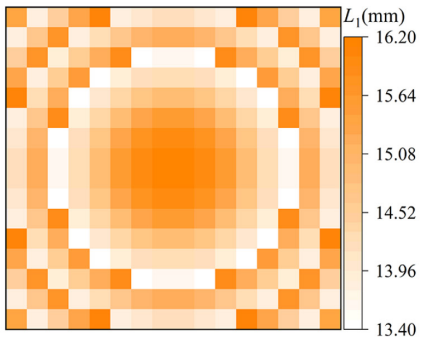

(b)
Fig. 7. (a) State distribution of the unit cell, (b) Value distribution of $L_{1}$.

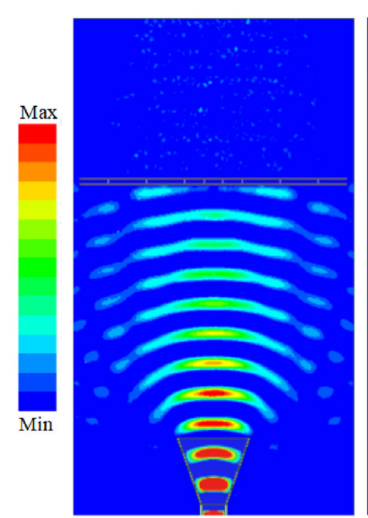

(a)

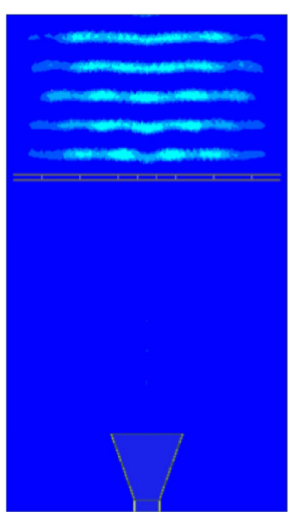

(b)
Fig. 8. Electric field distributions in xoz-plane at $8.5 \mathrm{GHz}$. (a) Magnitude of Ey. (b) Magnitude of Ex.

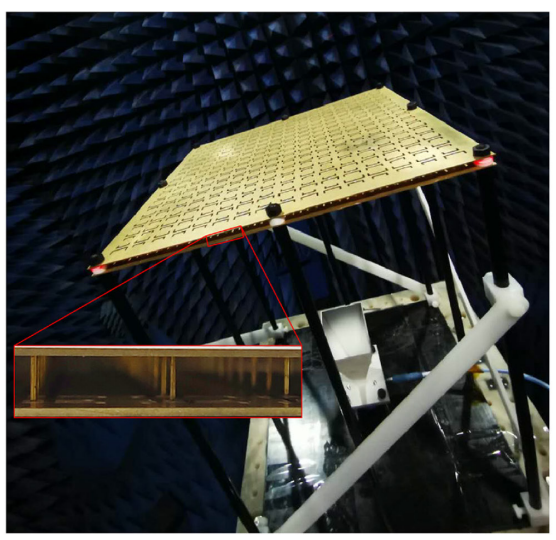

Fig. 9. Photograph of the fabricated TA prototypes.

$L_{1}$ is varied to provide additional phase shifts. The detailed distribution of the state of the unit cell in the TA panel and the value of $L_{1}$ are shown in Fig. 7. The electric field distribution in the near field around the TA is shown in Fig. 8. As shown, the TA converts quasi-spherical waves from the feed horn into outgoing quasi-plane waves propagating in the broadside direction with polarization conversion characteristic. To verify the radiation performance of the TA, a prototype of the proposed TA was fabricated, and a photo of the prototype under measurement is shown in Fig. 9. For the prototyping, the slots in the metal layers are processed by computer numerical control wire-cut machining. The metal post and the metal layers are connected by tin soldering, and the metal layers are polished to remove the excess solder. With the help of posts, the TA panel is rigid enough to be self-standing. Fig.10 compares the normalized co-polarization and cross-polarization radiation patterns between simulated and measured results in the E-plane. 


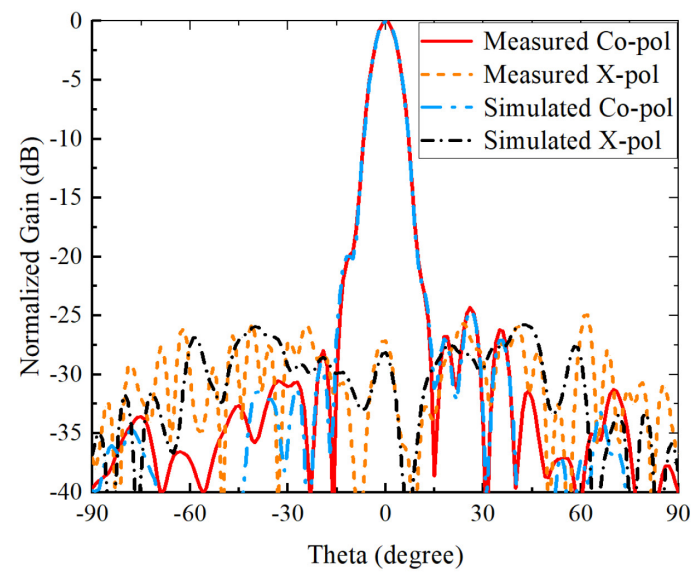

(a)

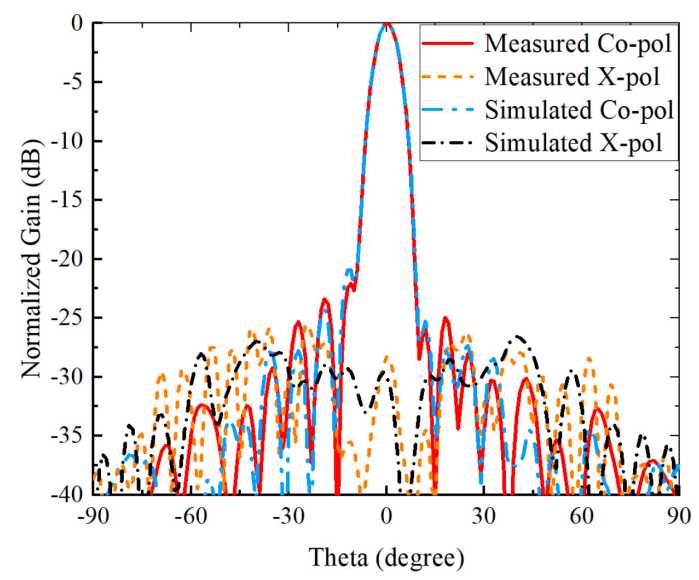

(b)

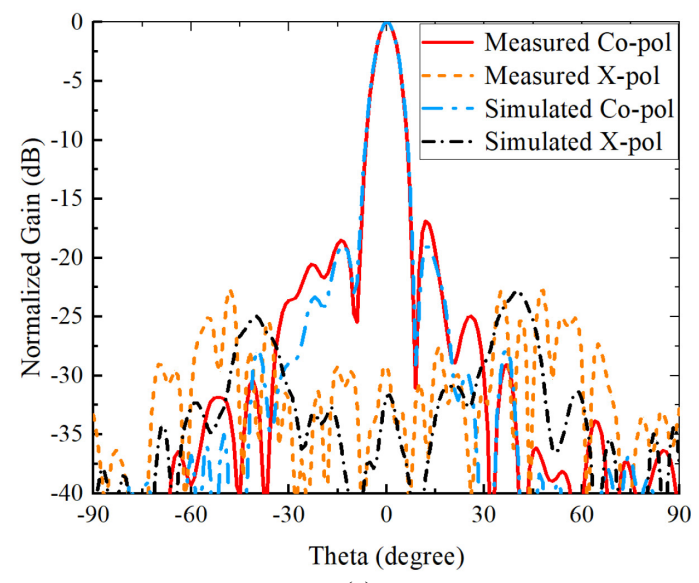

(c)

Fig. 10. Comparison of the measured and simulated radiation patterns. (a) E-plane at $8.2 \mathrm{GHz}$, (b) E-plane at $8.5 \mathrm{GHz}$, (c) E-plane at $9.5 \mathrm{GHz}$.

The frequencies are chosen as $8.2 \mathrm{GHz}, 8.5 \mathrm{GHz}$, and $9.5 \mathrm{GHz}$, where $8.2 \mathrm{GHz}$ and $9.5 \mathrm{GHz}$ represent the lowest and highest frequency in the $1-\mathrm{dB}$ gain bandwidth, respectively. There is a good agreement between the simulated and measured results. The normalized side-lobe levels are below $-15 \mathrm{~dB}$. The cross-polarization levels at the broadside direction are below $-25 \mathrm{~dB}$ in the E-plane within the 1-dB gain bandwidth. The simulated and measured gain and aperture efficiency of the TA are shown in Fig. 11. The aperture efficiency is calculated using the formula given in [20]. The measured results show that the array obtains a realized gain of $26 \mathrm{dBi}$ and the highest aperture

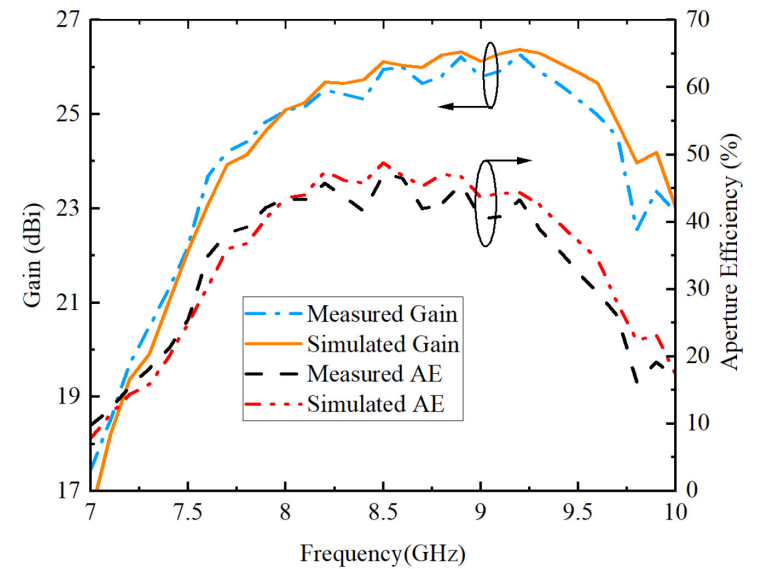

Fig. 11. Simulated and measured antenna gains and AE.

TABLE I

COMPARISON OF THE PRESENTED DESIGN WITH RePORTED METAL-ONLY TRANSMITARRAY DESIGNS

\begin{tabular}{cccccc}
\hline \hline Ref. & $\begin{array}{c}\text { No. of } \\
\text { layers }\end{array}$ & $\begin{array}{c}\text { Freq } \\
(\mathrm{GHz})\end{array}$ & $\begin{array}{c}\text { Aperture } \\
\text { diameter, } \\
\text { efficiency }\end{array}$ & $\begin{array}{c}\text { 1-dB } \\
\text { bandwidth }\end{array}$ & $\begin{array}{c}\text { 3-dB } \\
\text { bandwidth }\end{array}$ \\
\hline$[13]$ & 4 & 13.58 & $6.76 \lambda, 55.0 \%$ & $7.4 \%$ & $17.7 \%$ \\
{$[15]$} & 4 & 10.3 & $8.64 \lambda, 21.5 \%$ & $9.7 \%$ & -- \\
{$[16]$} & 3 & 10 & $6.4 \lambda, 55 \%$ & $15.5 \%$ & -- \\
{$[17]$} & 3 & 29.5 & $12.29 \lambda, 50.9 \%$ & -- & $11 \%$ \\
$\begin{array}{c}\text { This } \\
\text { work }\end{array}$ & $\mathbf{2}$ & $\mathbf{8 . 5}$ & $\mathbf{8 . 1 6} \lambda, \mathbf{4 7 . 6 \%}$ & $\mathbf{1 4 . 6 \%}$ & $\mathbf{2 4 . 3 \%}$ \\
\hline \hline
\end{tabular}

where $\lambda$ represents the wavelength of the center frequency in each design.

efficiency is at $8.5 \mathrm{GHz}(47.6 \%)$. The measured $1-\mathrm{dB}$ gain bandwidth and 3-dB gain bandwidth are 14.6\% (8.2-9.5 GHz) and $24.3 \%(7.6-9.7 \mathrm{GHz})$, respectively. Table I compares the presented design with the reported metal-only TAs. To achieve sufficient phase shift, the previously reported metal-only TAs use at least three layers. Compared to these reported metal-only TAs, the proposed metal-only unit cell has a two-layer structure and a lower profile but has wider bandwidth except for the design reported in [16]. The design reported in [16] has a little wider 1-dB gain bandwidth than the presented design, but the thickness of the TA panel is twice as the present design. Moreover, the proposed TA has improved mechanical robustness, low fabrication and assembling complexity.

\section{CONCLUSION}

In this letter, a novel two-layer metal-only wideband transmitarray with single linear polarization rotation is presented. The developed TA unit cell has multiple slots and shows a wide passband. Through simultaneously adjusting the length of the slots and changing the rotations of the slots on the bottom layer, a continuous $360^{\circ}$ phase shift is obtained with low insertion loss. The experimental results show the prototype exhibits wide 1-dB and 3-dB gain bandwidth. The presented dual-layer metal-only TA simplifies the fabrication and has good mechanical robustness. Compared to existing metal-only TA designs, the TA panel of the present design has fewer layers but with wide operation bandwidth and high efficiency. 


\section{REFERENCES}

[1]. K.-W. Lam, S.-W. Kwok, Y. Hwang, and T. K. Lo, "Implementation of transmitarray antenna concept by using aperture-coupled microstrip patches," Proc. Asia-Pacific Microwave APMC'97, 1997, vol. 1, pp. 433-436.

[2]. Q. Luo, S. Gao, M. Sobhy and X. Yang, "Wideband Transmitarray With Reduced Profile," IEEE Antennas Wireless Propag. Lett, vol. 17, no. 3, pp. 450-453, March 2018.

[3]. A. H. Abdelrahman, P. Nayeri, A. Z. Elsherbeni and F. Yang, "Single-Feed Quad-Beam Transmitarray Antenna Design," IEEE Trans. Antennas Propag., vol. 64, no. 3, pp. 953-959, March 2016.

[4]. H. Kaouach, L. Dussopt, J. Lanteri, T. Koleck and R. Sauleau, "Wideband Low-Loss Linear and Circular Polarization Transmit-Arrays in V-Band," IEEE Trans. Antennas Propag., vol. 59, no. 7, pp. 2513-2523, July 2011.

[5]. K. T. Pham, R. Sauleau, E. Fourn, F. Diaby, A. Clemente, and L. Dussopt, "Dual-Band Transmitarrays With Dual-Linear Polarization at Ka-Band," IEEE Trans. Antennas Propag., vol. 65, no. 12, pp. 7009-7018, Dec. 2017.

[6]. Y.-M. Cai, K. Li, W. Li, S. Gao, Y. Yin, L. Zhao, and W. Hu, "Dual-Band Circularly Polarized Transmitarray With Single Linearly Polarized Feed," IEEE Trans. Antennas Propag., vol. 68, no. 6, pp. 5015-5020, June 2020.

[7]. F. F. Manzillo, A. Clemente, and J. L. González-Jiménez, "High-gain D-band Transmitarrays in Standard PCB Technology for Beyond-5G Communications," IEEE Trans. Antennas Propag., vol. 68, no. 1, pp. 587-592, Jan. 2020.

[8]. Y. Wang, S. Xu, F. Yang, and M. Li, "A Novel 1-bit Wide-angle Beam Scanning Reconfigurable Transmitarray Antenna Using an Equivalent Magnetic Dipole Element," IEEE Trans. Antennas Propag., vol. 68, no. 7, pp. 5691-5695, July 2020.

[9]. F. Qin, S. Gao, W. Cheng, Y. Liu, H. Zhang, and G. Wei, "A High-Gain Transmitarray for Generating Dual-Mode OAM Beams," IEEE Access, vol. 6, pp. 61006-61013, 2018.

[10]. Y.-M Cai, W. Li, K. Li, S. Gao, Y. Yin, L. Zhao, and W. Hu, "A Novel Ultrawideband Transmitarray Design Using Tightly Coupled Dipole
Elements," IEEE Trans. Antennas Propag., vol. 67, no. 1, pp. 242-250, Jan. 2019.

[11]. A. H. Abdelrahman, A. Z. Elsherbeni, and F. Yang, "Transmission Phase Limit of Multilayer Frequency-Selective Surfaces for Transmitarray Designs," IEEE Trans. Antennas Propag., vol. 62, no. 2, pp. 690-697, Feb. 2014.

[12]. A. H. Abdelrahman, A. Z. Elsherbeni and F. Yang, "Transmitarray Antenna Design Using Cross-Slot Elements With No Dielectric Substrate," IEEE Antennas Wireless Propag. Lett., vol. 13, pp. 177-180, 2014.

[13]. G. Liu, H.-j. Wang, J.-s. Jiang, F. Xue, and M. Yi, "A High-Efficiency Transmitarray Antenna Using Double Split Ring Slot Elements," IEEE Antennas Wireless Propag. Lett., vol. 14, pp. 1415-1418, 2015.

[14]. R. Y. Wu, Y. B. Li, W. Wu, C. B. Shi, and T. J. Cui, "High-Gain Dual-Band Transmitarray," IEEE Trans. Antennas Propag., vol. 65, no. 7, pp. 3481-3488, July 2017.

[15]. J. Zhao, T. Li, H. Li, X. Yang, Y. Zhou, W. Mao, H. Wang, B. Hu, H. Zou, Q. Liu, "A Novel Planar Mode-Transducing Antenna Based on Metal-Only Transmitarray Surfaces," IEEE Trans. Antennas Propag., vol. 67, no. 6, pp. 3762-3773, June 2019.

[16]. B. Rahmati, H. R. Hassani, "High-Efficient Wideband Slot Transmitarray Antenna," IEEE Trans. Antennas Propag., vol. 63, no. 11, pp. 5149-5155, Nov. 2015.

[17]. K. T. Pham, A. Clemente, E. Fourn, F. Diaby, L. Dussopt, and R. Sauleau, "Low-Cost Metal-Only Transmitarray Antennas at Ka-Band," IEEE Antennas Wireless Propag. Lett., vol. 18, no. 6, pp. 1243-1247, June 2019 .

[18]. W. An, S. Xu, F. Yang, and M. Li, "A Double-Layer Transmitarray Antenna Using Malta Crosses With Vias," IEEE Trans. Antennas Propag., vol. 64, no. 3, pp. 1120-1125, March 2016.

[19]. X. Yi, T. Su, X. Li, B. Wu, and L. Yang, "A Double-Layer Wideband Transmitarray Antenna Using Two Degrees of Freedom Elements Around 20 GHz," IEEE Trans. Antennas Propag., vol. 67, no. 4, pp. 2798-2802, April 2019.

[20]. C. A. Balanis, Antenna theory analysis and design, 3rd ed. New York, U.S.: Willey, 2005. 